\title{
THE PROCESSES OF HOMEOSTASIS, CHEMOTAXIS AND ORGANIC AND INORGANIC RESPONSE ARE SIGNIFICANTLY UP-REGULATED DURING SHORT-TERM ORAL MUCOSAL CELLS IN VITRO CULTIVATION
}

Blanka Borowiec ${ }^{1,2}$, Sylwia Ciesiółka ${ }^{1}$, Krzysztof Janowicz ${ }^{2,3}$, Piotr Celichowski ${ }^{1}$, Artur Bryja ${ }^{2}$, Lisa Moncrieff ${ }^{1,3}$, Greg Hutchings ${ }^{2,3}$, Claudia Dompe ${ }^{1,3}$, Bartosz Kempisty ${ }^{1,2,4,7}$ Jamil A. Shibli $^{5}$, Paul Mozdziak $^{6}$, Karolina Gerreth ${ }^{8}$, Marta Dyszkiewicz-Konwińska ${ }^{2,9}$

\begin{abstract}
Mucous membranes appear in various parts of the whole body performing similar functions. However, they differ based on where the mucosa is located. It functions as a barrier in such systems as: respiratory, urogenital and digestive. In this study we will be focusing strictly on the oral mucosa. Keratinocytes and fibroblasts, which mainly form the structure of the oral mucosa, are subjected to numerous factors. Being one of the million parts that build the animal organism, they are involved in various processes. In this study, we will try to confirm that in the in vitro culture of oral mucosa cells, the expression of our selected genes undergoes significant changes which are tied to such processes as: homeostasis, chemotaxis and organic/inorganic response of the organism. For this study, 20 pubertal crossbred Landrace gilts were used. After slaughter, samples of buccal pouch mucosa were obtained and transported to the laboratory. The excised tissue was prepared and processed due to protocols. The final pellet was resuspended in supplemented DMEM. Once the cultures attained 70-80\% confluency, they were passaged. Total RNA from each pooled sample was subjected to two rounds of sense cDNA amplification. The cDNA was processed on microarrays. Analysis of the scanned arrays was performed. The files were imported into downstream data analysis software. The DAVID analysis showed that differently expressed genes belongs to 56 Gene ontology groups. In this paper we focused on "cellular divalent inorganic cation homeostasis", "chemical homeostasis", "chemotaxis", "homeostatic process" and "response to organic substance" GO BP terms. These sets of genes were subjected to hierarchical clusterization procedure. In summary, the data we collected showed primarily changes in gene expression that occurred in the thirty-day cell culture of oral mucosa tissue. We assume that indicated genes could be new gene markers for studied processes.
\end{abstract}

Running title: Homeostasis in oral mucosa cells

Keywords: oral mucosa cells, homeostasis, chemotaxis, in vitro culture

\footnotetext{
${ }^{1}$ Department of Histology and Embryology, Poznań University of Medical Sciences, Poznań, Poland

${ }^{2}$ Department of Anatomy, Poznań University of Medical Sciences, Poznań, Poland

${ }^{3}$ The School of Medicine, Medical Sciences and Nutrition, University of Aberdeen, Aberdeen, UK

${ }^{4}$ Department of Obstetrics and Gynecology, University Hospital and Masaryk University, Brno, Czech Republic

${ }^{5}$ Department of Periodontology and Oral Implantology, Dental Research Division, University of Guarulhos, Guarulhos, SP, Brazil

${ }^{6}$ Physiology Graduate Program, North Carolina State University, Raleigh, USA

${ }^{7}$ Department of Veterinary Surgery, Institute of Veterinary Medicine, Nicolaus Copernicus University, Toruń, Poland

${ }^{8}$ Department of Risk Group Dentistry, Chair of Pediatric Dentistry, Poznań University of Medical Sciences, Poznań, Poland

${ }^{9}$ Department of Biomaterials and Experimental Dentistry, Poznań University of Medical Sciences, Poznań, Poland

* Correspondence: mdyszkiewicz@ump.edu.pl

Full list of author information is available at the end of article
} 


\section{Introduction}

Mucous membrane is found in different areas of the body performing similar functions. However, they differ mainly based on their location . Mucous membrane functions as a barrier in such systems as: respiratory, urogenital and digestive. In this study we will be focusing strictly on the oral mucosa. The oral cavity is coated with a unceasing layer of mucous membrane type II [1]. This tissue is adjacent to the pharyngeal mucosa and the skin at the vermillion border of the lips. It is mainly of ectodermal origin and consists of layers of squamous epithelium. Like the skin, the mucosa serves as a protective barrier against mechanical and microbiological injuries, it is designed to receive stimuli such as touch, pain and temperature, and to serve as a secreting organ. Its permeability creates great conditions for the absorption of microparticles, such as various types of medications [2]. Keratinocytes and fibroblasts, which mainly form the structure of the oral mucosa, are subjected to numerous factors. Being one of the million parts that build the animal organism, they are involved in various processes. One of this biological phenomena is homeostasis. By this term we understand any self-regulating process by which biological systems incline to maintain stability while adapting to conditions that are most advantageous for survival [3]. This is an extremely complicated process, considering how many aspects creating it and how many elements are involved. With regard to the oral cavity, homeostasis involves balance between keratinoblasts, keratinocytes and fibroblasts. Keeping this equilibrium is crucial for morphological modification of oral mucosa [4]. As highly active elements, these cells show strong chemotaxis. For example, activated keratinocytes express surface receptors for cytokines, chemokines and opioids [5-7]. Morphological status of the oral mucosa significantly affects the metabolism and biochemistry of this tissue[8]. Based on the available literature and knowledge, we have developed an effective culture system for mucosal keratinocytes and fibroblasts, which are previously isolated from the oral mucosa of Sus scrofa f. domestica. In addition, the European Medicines Agency (EMA) still requires the use of large animals in comparative tests as a model similar to humans. In this case, it was necessary to use this particular animal model due to similarities :physiological, anatomical and nutritional to humans. Recognized ontological groups, whose representatives are genes with significantly reduced or increased expression, suggest that oral mucosa cells are involved in homeostasis and control of the body's metabolism. Microarray approach enables us to learn about new metabolic pathways in which known and previously unknown genes are involved. Our previous microarray studies on gene expression in cultured cells have shown significant changes in the transcriptomic profile. And it was closely linked to which day of growth cells were currently in [9]. It is also confirmed, that during cultivation of the oral mucosa cells, the cells undergo significant differentiation and visible proliferation [8].

In this study, we will try to confirm that in the in vitro culture of oral mucosa, the expression of our selected genes undergo significant changes which are tied to such processes as: homeostasis, chemotaxis and organic/ingorganic response of the organism. We will analyze whether the increased or decreased expression of selected genes is in line with the available literature and we will try to explain why the expression of a given gene changes at a specific time frame in the cell culture.

\section{Materials and Methods \\ Animals}

For this study, a total of 20 pubertal crossbred Landrace gilts bred on commercial local farm were used. They had a mean age of 155 days (range 140 - 170 days), and the mean weight was $100 \mathrm{~kg}$ (95$120 \mathrm{~kg}$ ). All of the animals were housed under identical conditions and fed the same forage (depending on age and reproductive status). The experiments were approved by Local Ethic Committee.

\section{Cell isolation and culture}

After slaughter, samples of buccal pouch mucosa were obtained within $40 \mathrm{~min}$ and transported to the laboratory. The excised tissue was washed twice in Dulbecco's phosphate buffered saline (D-PBS) (137 $\mathrm{mM} \mathrm{NaCl}, 27 \mathrm{mM} \mathrm{KCl}, 10 \mathrm{mM} \mathrm{Na} 2 \mathrm{HPO} 4,2 \mathrm{mM} \mathrm{KH}-$ 2P04, pH 7.4). The surface of the buccal pouch was surgically removed using sterile surgical blades. The tissue fragments were incubated with $0.05 \%$ collagenase I (Sigma Aldrich, Madison, USA) for 40 min at $38{ }^{\circ} \mathrm{C}$ in a shaking water bath and then were treated witch 0.5\% Trypsin/EDTA (Cascade Biologics, Portland, USA) for $10 \mathrm{~min}$. The cell suspension obtained from this digestion was filtered through mesh to remove non-dissociated tissue fragments. Isolated cells were washed three times by centrifugation (10 min at $200 \mathrm{~g}$ ) with Dulbecco's modified Eagle's medium (DMEM; Sigma Aldrich, Madison, USA) supplemented with gentamicin $(20 \mu \mathrm{g} / \mathrm{mL})$ and $0.1 \%$ BSA. The final cell pellet was resuspended in DMEM supplemented with $10 \%$ fetal calf serum (FCS; Sigma Aldrich, Madison, USA) and $10 \mathrm{U} /$ $\mathrm{mL}$ penicillin G, $10 \mathrm{mg} / \mathrm{mL}$ streptomycin, and $25 \mu \mathrm{g} /$ $\mathrm{mL}$ amphotericin B. Cell viability was 90 to $95 \%$ as determined by trypan blue staining (Sigma Aldrich, Madison, USA). The cells were cultured at $38.5{ }^{\circ} \mathrm{C}$ in a humidified atmosphere of $5 \% \mathrm{CO}_{2}$. Once the keratinocyte cultures attained 70-80\% confluency, they were passaged by washing with PBS, digested with 0.025\% Trypsin/EDTA (Cascade Biologics, Portland, USA), neutralized by a $0.0125 \%$ trypsin inhibitor (Cascade Biologics, Portland, USA), centrifuged, and resuspended at a seeding density of $2 \times 104$ cells $/ \mathrm{cm}^{2}$. The culture medium was changed every three days. 


\section{Microarray expression analysis and statistics}

Total RNA (100 ng) from each pooled sample was subjected to two rounds of sense cDNA amplification (Ambion $₫$ WT Expression Kit). The obtained cDNA was used for biotin labeling and fragmentation by Affymetrix GeneChip $₫$ WT Terminal Labeling and Hybridization (Affymetrix). Biotin-labeled fragments of cDNA $(5.5 \mu \mathrm{g})$ were hybridized to the Affymetrix $\AA$ Porcine Gene 1.1 ST Array Strip $\left(48^{\circ} \mathrm{C} / 20 \mathrm{~h}\right)$. Microarrays were then washed and stained according to the technical protocol using the Affymetrix GeneAtlas Fluidics Station. The array strips were scanned employing Imaging Station of the GeneAtlas System. Preliminary analysis of the scanned chips was performed using Affymetrix GeneAtlasTM Operating Software. The quality of gene expression data was confirmed according to the quality control criteria provided by the software. The obtained CEL files were imported into downstream data analysis software.

All of the presented analyses and graphs were performed using Bioconductor and R programming languages. Each CEL file was merged with a description file. In order to correct background, normalize, and summarize results, we used the Robust Multiarray Averaging (RMA) algorithm. To determine the statistical significance of the analyzed genes, moderated t-statistics from the empirical Bayes method were performed. The obtained p-value was corrected for multiple comparisons using Benjamini and Hochberg's false discovery rate. The selection of significantly altered genes was based on a p-value beneath 0.05 and expression higher than two fold.

Differentially expressed genes were subjected to selection by examination of genes involved in cell mi- gration regulation. The differentially expressed gene list (separated for up- and down-regulated genes) was uploaded to DAVID software (Database for Annotation, Visualization and Integrated Discovery)[10].

Subsequently we analyzed the interaction between the genes belonging to chosen GO terms with GOplot package (Walter, Wencke, Fatima Sanchez-Cabo, and Mercedes Ricote. GOplot: an R package for visually combining expression data with functional analysis. Bioinformatics (2015): btv300.)

Finally interactions between differentially expressed genes/proteins belonging to the chosen GO terms were investigated by STRING10 software (Search Tool for the Retrieval of Interacting Genes) (von Mering et al., 2005). The list of gene names was used as a query for an interaction prediction. The search criteria were based on co-occurrences of genes/proteins in scientific texts (text mining), co-expression, and experimentally observed interactions. The results of such analyses generated a gene/protein interaction network where the intensity of the edges reflected the strength of the interaction score.

\section{Ethical approval}

The research related to animal use has been complied with all the relevant national regulations and instructional policies for the care and use of animals. Bioethical Committee approval no. 32/2012, 30.06.2012.

\section{Results}

Whole transcriptome profiling by affymetrix microarray, allow as analyzing gene expression changes between 7, 15 and 30 days of buccal pouch mucosa cells culture. By Affymetrix ${ }^{\circledR}$ Porcine Gene 1.1
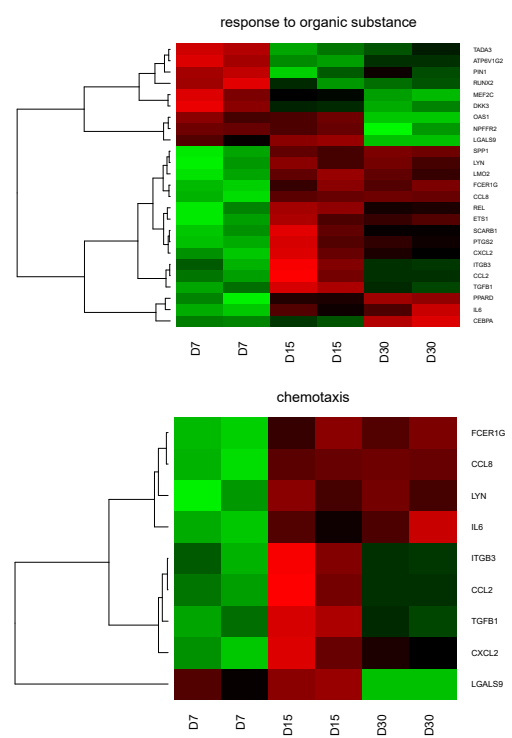
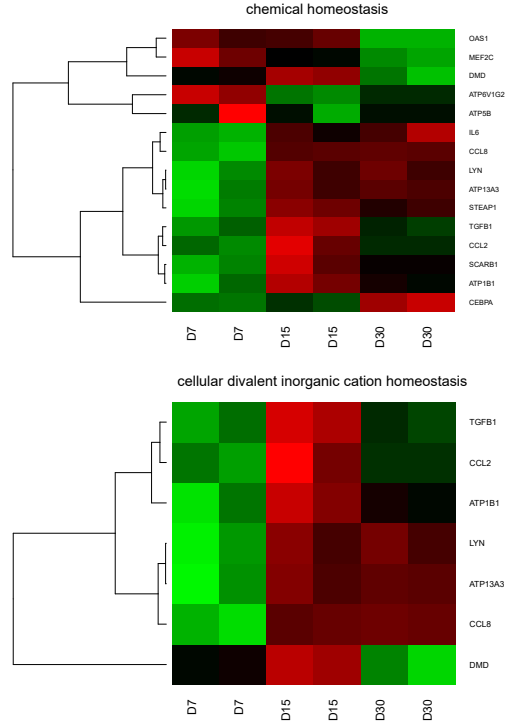

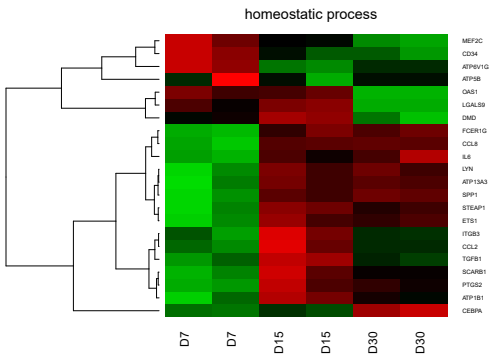

FIGURE 1 Heat map representation of differentially expressed genes belonging to the "cellular divalent inorganic cation homeostasis", "chemical homeostasis", "chemotaxis", "homeostatic process" and "response to organic substance" GO BP terms. Arbitrary signal intensity acquired from microarray analysis is represented by colours (green, higher; red, lower expression). Log2 signal intensity values for any single gene were resized to Row Z-Score scale (from -2, the lowest expression to +2 , the highest expression for single gene) 
TABLE 1 Gene symbols, Entrez gene IDs, ratio and corrected P values of studied genes

\begin{tabular}{|c|c|c|c|c|c|c|}
\hline GENE SYMBOL & GENE ID & RATIO D7/D15 & RATIO D7/D30 & P VAL D7/D15 & P VAL D7/D30 & MEAN RATIO \\
\hline SPP1 & 397087 & -10.87016565 & -14.2538617 & 0.016271204 & 0.023019379 & -12.56201368 \\
\hline CCL8 & 100302703 & -9.10436286 & -9.846671298 & 0.009909162 & 0.002855033 & -9.475517079 \\
\hline CXCL2 & 414904 & -6.305216475 & -2.763765187 & 0.028210571 & 0.100060673 & -4.534490831 \\
\hline PTGS2 & 397590 & -5.663189033 & -3.05066486 & 0.033272153 & 0.086182814 & -4.356926947 \\
\hline ATP13A3 & 100522900 & -3.576872002 & -3.427204735 & 0.033844567 & 0.040686213 & -3.502038369 \\
\hline PPARD & 397671 & -2.416675019 & -4.005391116 & 0.052006062 & 0.023501509 & -3.211033067 \\
\hline ATP1B1 & 396898 & -3.743863827 & -2.010447374 & 0.031118493 & 0.123030328 & -2.8771556 \\
\hline CCL2 & 397422 & -3.929361381 & -1.450779929 & 0.040743821 & 0.404390916 & -2.690070655 \\
\hline LYN & 100152890 & -2.52053573 & -2.454535196 & 0.034636492 & 0.04233411 & -2.487535463 \\
\hline REL & 100525104 & -3.00471739 & -1.96555405 & 0.023427948 & 0.05637387 & -2.48513572 \\
\hline FCER1G & 397406 & -2.351156762 & -2.404588058 & 0.026835676 & 0.023565415 & -2.37787241 \\
\hline ETS1 & 100302363 & -2.513407982 & -2.150521983 & 0.031118493 & 0.047472133 & -2.331964982 \\
\hline SCARB1 & 397018 & -2.623056585 & -1.681749941 & 0.034636492 & 0.134327075 & -2.152403263 \\
\hline STEAP1 & 397573 & -2.349620022 & -1.88662936 & 0.026835676 & 0.044202404 & -2.118124691 \\
\hline ITGB3 & 397063 & -2.917250333 & -1.311025215 & 0.038898003 & 0.421388587 & -2.114137774 \\
\hline IL6 & 399500 & -1.793617663 & -2.249334565 & 0.088464766 & 0.044202404 & -2.021476114 \\
\hline LMO2 & 100512825 & -2.0687671 & -1.856369805 & 0.026835676 & 0.034894667 & -1.962568452 \\
\hline TGFB1 & 397078 & -2.253272341 & -1.226316922 & 0.015016424 & 0.216600729 & -1.739794631 \\
\hline CEBPA & 397307 & -1.147965724 & -2.175053003 & 0.320782294 & 0.017618993 & -1.661509363 \\
\hline DMD & 497636 & -1.603674593 & 1.631209228 & 0.072461804 & 0.068161696 & 0.013767318 \\
\hline LGALS9 & 396972 & -1.572710066 & 2.910882324 & 0.1398614 & 0.023501509 & 0.669086129 \\
\hline ATP5B & 100157156 & 1.122331171 & 1.073246663 & 0.482886753 & 0.62979588 & 1.097788917 \\
\hline NPFFR2 & --- & 1.044570266 & 2.010006004 & 0.826996209 & 0.026707771 & 1.527288135 \\
\hline OAS1 & --- & 1.023318222 & 2.255519223 & 0.911552638 & 0.023019379 & 1.639418722 \\
\hline PIN1 & 100512827 & 2.022694206 & 1.581852096 & 0.04252503 & 0.117593514 & 1.802273151 \\
\hline DKK3 & 664653 & 1.778040065 & 2.362675908 & 0.0466037 & 0.023501509 & 2.070357987 \\
\hline ATP6V1G2 & 100152358 & 2.346891412 & 1.83072396 & 0.013032038 & 0.023501509 & 2.088807686 \\
\hline TADA3 & 100157464 & 2.510073124 & 1.977935459 & 0.015016424 & 0.024934184 & 2.244004291 \\
\hline MEF2C & 733590 & 1.801279355 & 3.223918682 & 0.081198998 & 0.023501509 & 2.512599019 \\
\hline RUNX2 & 100737965 & 2.658456436 & 2.666226873 & 0.04252503 & 0.047472133 & 2.662341655 \\
\hline CD34 & 397160 & 2.363067501 & 3.093363256 & 0.052291352 & 0.034894667 & 2.728215378 \\
\hline
\end{tabular}

ST Array Strip we examined expression of 12257 transcripts. Genes with fold change higher than abs (2) and wit corrected p value lower than 0.05 were considered as differentially expressed. This set of genes consists of 130 different transcripts. The first detailed analysis based on GO BP the identification of differentially expressed genes belonging to the significantly enrichment GO BP terms.

DAVID (Database for Annotation, Visualization and Integrated Discovery) software was used for extraction of gene ontology biological process term (GO BP) that contains differently expressed transcripts. Up and down regulated gene sets were subjected to DAVID searching separately and only gene sets where adj. $p$ value were lower than 0.05 were selected. The DAVID software analysis showed that differently expressed genes belongs to 56 Gene ontology groups. In this paper we focused on "cellular divalent inorganic cation homeostasis", "chemical homeostasis", "chemotaxis", "homeostatic process" and "response to organ- ic substance" GO BP terms. These sets of genes were subjected to hierarchical clusterization procedure and presented as heatmaps (Fig. 1). The gene symbols, fold changes in expression, Entrez gene IDs and corrected $\mathrm{p}$ values of that genes were shown in table $\mathbf{1}$.

Moreover in Gene Ontology database genes that formed one particular GO group can also belong to other different GO term categories. By this reason we explore the gene intersections between selected GO BP terms. The relation between those GO BP terms was presented as well chart and Venn diagram (Fig. 2) as well as heatmap (Fig. 3).

STRING-generated interaction network among differentially expressed genes belonging to each of selected GO BP terms. Using such prediction method provided us molecular interaction network formed between protein products of studied genes (Fig. 4). The last figure (Fig. 5) is showing the differences in occurrence of differently expressed genes in frequency from 1 to 5 . 
D15/D7

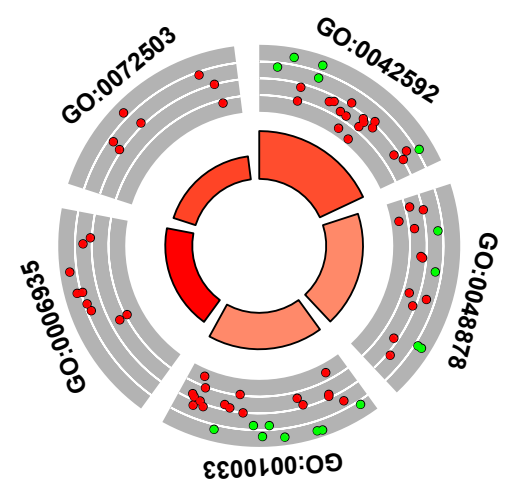

$\underbrace{\text { z-score }}_{\text {decreasing }}$ increasing $\quad$ logFC
D30/D7

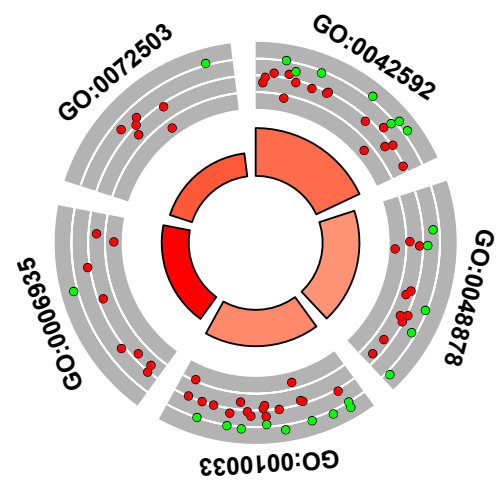

D30/D15

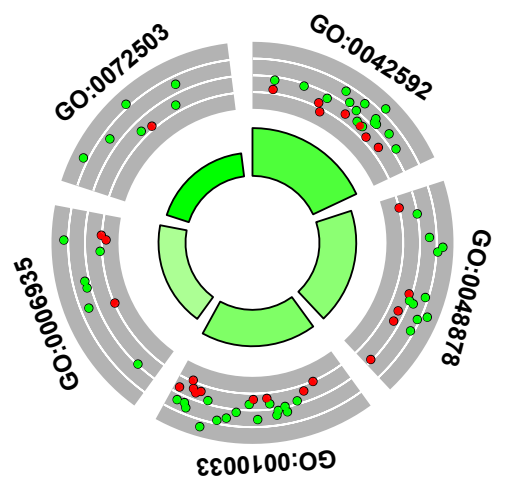

\begin{tabular}{|c|c|}
\hline ID & Description \\
\hline GO:0042592 & homeostatic process \\
\hline GO:0048878 & chemical homeostasis \\
\hline GO:0010033 & response to organic substance \\
\hline GO:0006935 & chemotaxis \\
\hline GO:0072503 & cellular divalent inorganic cation homeostasis \\
\hline
\end{tabular}

FIGURE 2 The circle plot showing the differently expressed genes and z-score of the "cellular divalent inorganic cation homeostasis", "chemical homeostasis", "chemotaxis", "homeostatic process" and "response to organic substance" GO BP terms. The outer circle shows a scatter plot for each term of the fold change of the assigned genes. Green circles display up- regulation and red ones down- regulation. The inner circle shows the z-score of each GO BP term. The width of the each bar corresponds to the number of genes within GO BP term and the color corresponds to the z-score

\section{Discussion}

The oral mucosa is characterized by intensive and constant changes in its morphology and biochemistry. The basis for the proper functioning of the mucosa is the balance between keratinoblasts, keratinocytes and fibroblasts. This balance can be influenced by many external factors, such as environmental changes, mechanical stress, and drug delivery [8]. To characterize oral soft tissues comportment, we shall use mucosal biomechanical parameters as reference. Thanks to this, we will be able to refer to clinical applications such as pressure-pain thresholds, stimuli for tissue remodeling or testing new drugs and dressings [11]. Epithelial regeneration processes form the basis of stem cell and oral cancer research. These synthetically cultured tissue analogies are used in transplant surgery to treat numerous tissue dysfunctions in areas such as the urethra or esophagus $[12,13]$.

However, to apply the epithelial properties listed above into the practice, we first need to know their molecular background. We chose genes with noticeable expression changes and from that group we have discussed 10 selected genes with the highest and lowest expression from the whole pool studied. The control sample in this study was day 7 of the culture. The 15 th and 30th days were compared to this sample.

The CD34 gene showed the highest expression in relation to the seventh day. Single-pass membrane protein encoded by this gene, may play a role in the attachment of stem cells to the bone marrow extracellular matrix or to stromal cells. This protein is extremely phosphorylated and glycosylated by protein kinase C [14].

RUNX2 encodes protein which is essential for osteoblastic differentiation and skeletal morphogenesis. It also acts as a scaffold for nucleic acids and regulatory factors participating in skeletal gene expression. Core binding factor (CBF) binds to the core site, 5'-PYGPYGGT-3', of a number of enhancers and promoters, including murine leukemia virus, polyomavirus enhancer, T-cell receptor enhancers, osteocalcin and osteopontin [15]. In turn, MEF2C refers to myogenesis. The protein encoded by this gene, MEF2 polypeptide $\mathrm{C}$, has both trans-activating and DNA binding activities. This protein may play a role in keeping the differentiated state of muscle cells. Mutations and deletions at this locus have been associated with severe cognitive disability, cerebral malformations and epilepsy. It also controls cardiac morphogenesis and myogenesis, and is likewise involved in vascular development. Enhances transcriptional activation mediated by SOX18 $[16,17]$. The next place in the table was placed by the TADA3 gene. DNA-binding transcriptional activator proteins encoded by this gene, increases the rate of transcription. It happens when the transcriptional machinery bound to the basal promoter in conjunction with adaptor proteins [18]. The protein encoded by this gene is also a component 


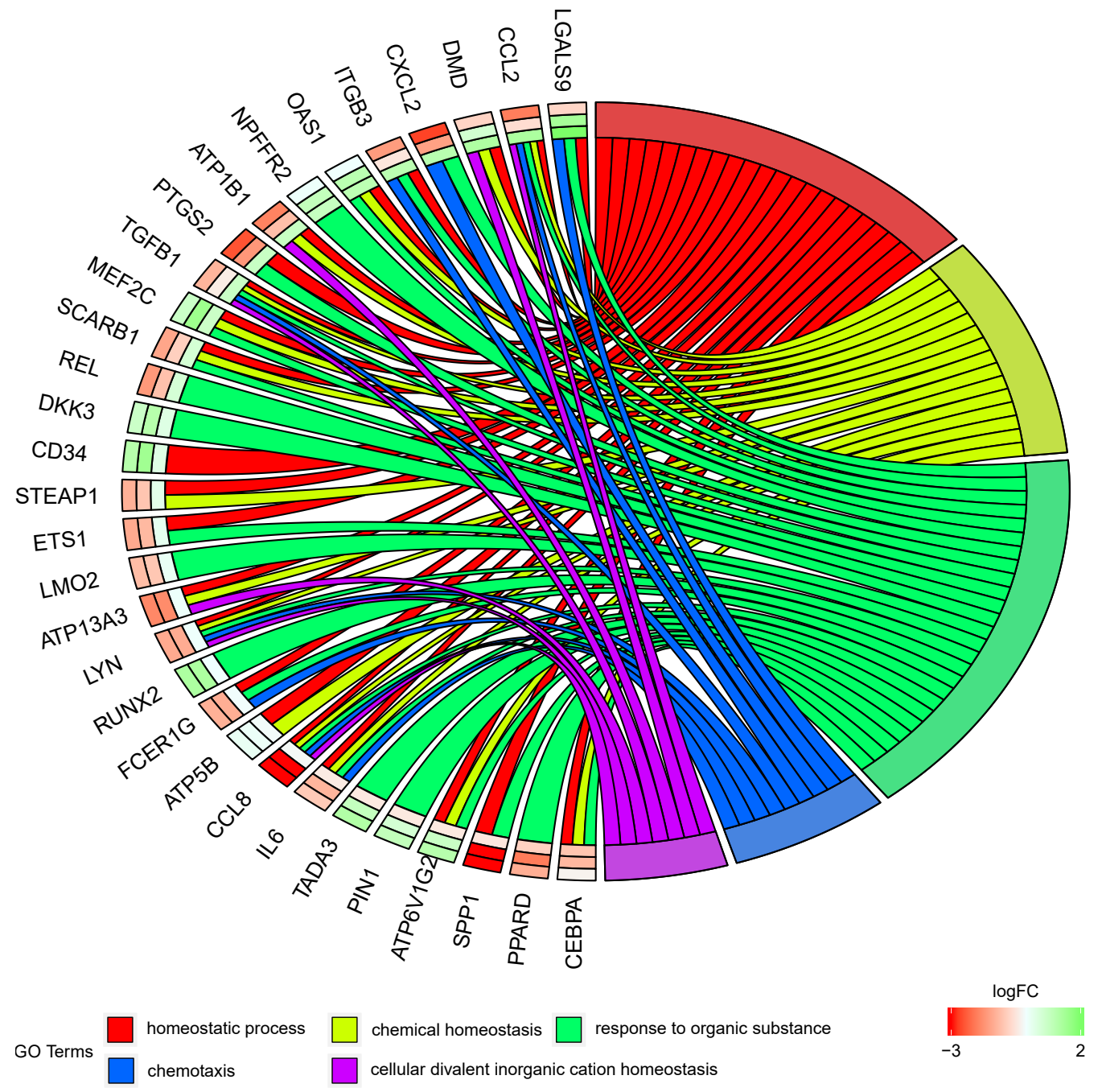

FIGURE 3 The representation of the mutual relationship of differently expressed genes "cellular divalent inorganic cation homeostasis", "chemical homeostasis", "chemotaxis", "homeostatic process" and "response to organic substance" GO BP terms. The ribbons indicate which gene belongs to which categories. The middle circle represents logarithm from fold change (LogFC) between D15/D7, D30/D7 and D30/D15 respectively. The color of each block corresponds to the LogFC of each gene (green - upregulated, red - downregulated). The genes were sorted by logFC from most to least changed gene

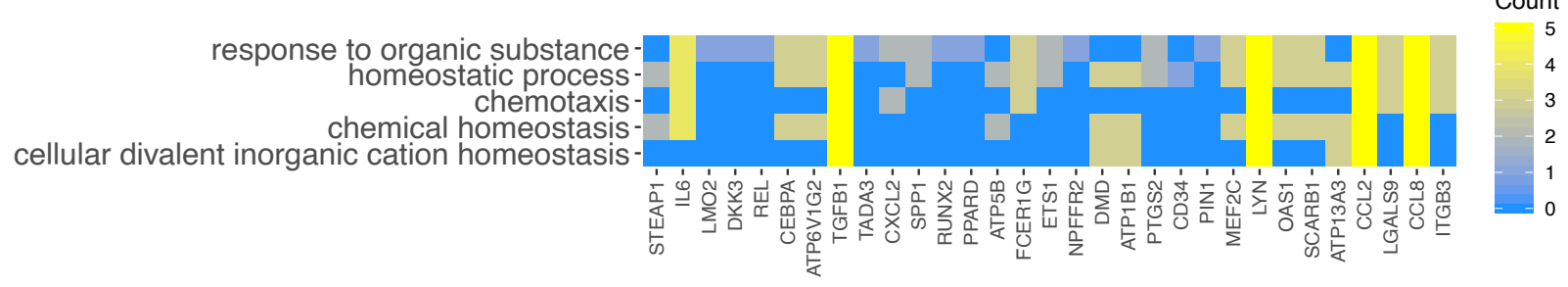

FIGURE 5 STRING-generated interaction occurrence differently expressed genes that belongs to the "cellular divalent inorganic cation homeostasis", "chemical homeostasis", "chemotaxis", "homeostatic process" and "response to organic substance" GO BP terms. The intensity of the edges reflects the interaction. The explanation of each symbol is included on the legend included in figure

of the histone acetyl transferase (HAT) coactivator complex. It plays a crucial role in chromatin modulation, cell cycle progression and cellular response to DNA damage [19]. The next 4 genes appeared in the group "response to organic substance". ATP6V1G2 is the gene that encodes a component of vacuolar ATPase (V-ATPase) [20]. It is multi-subunit enzyme that facilitates acidification of intracellular compartments of eukaryotic cells. It is necessary for such intracellular processes as protein sorting and activation, synaptic vesicle proton gradient generation and receptor-mediated endocytosis. It is 


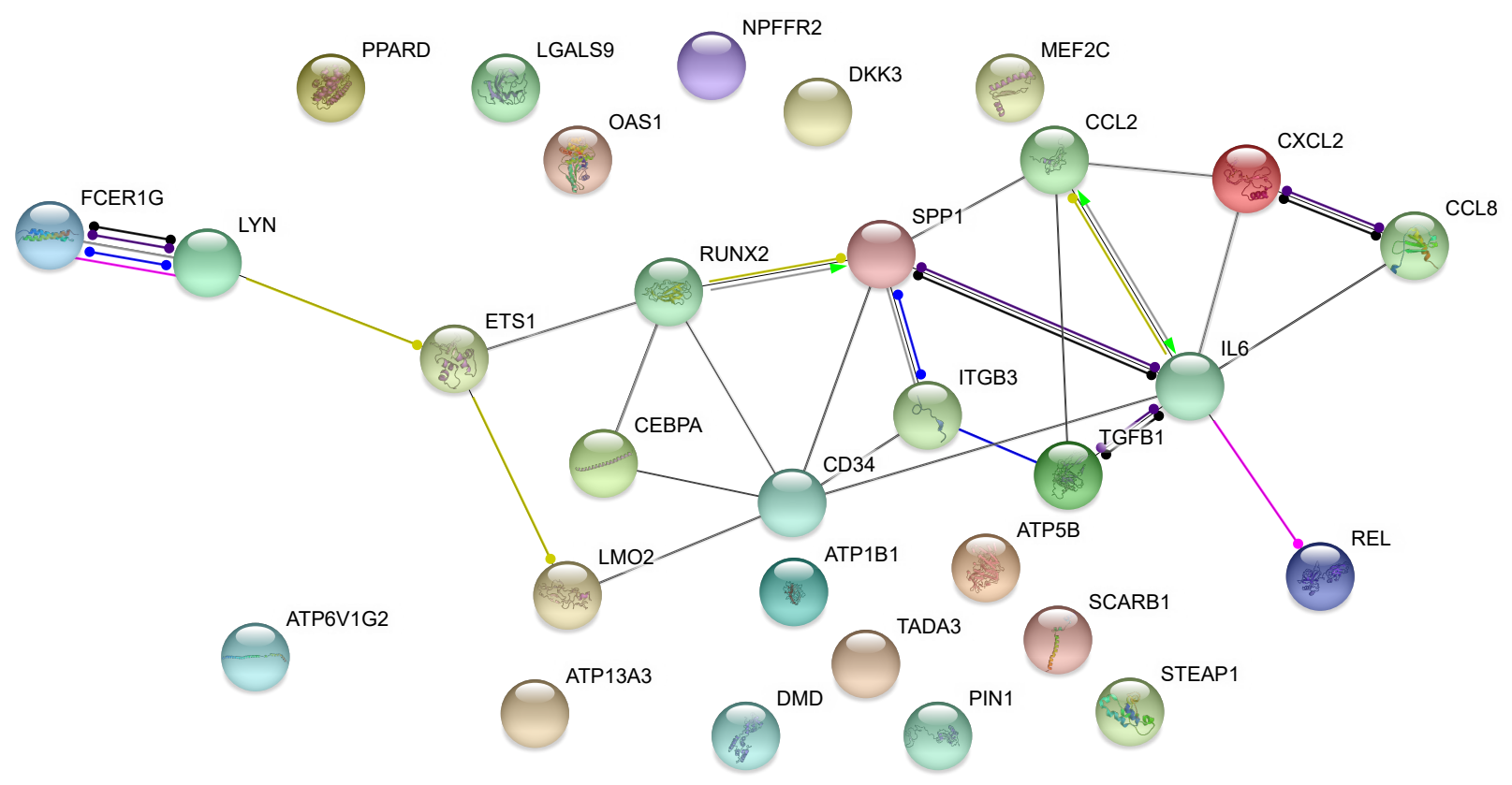

Action Types
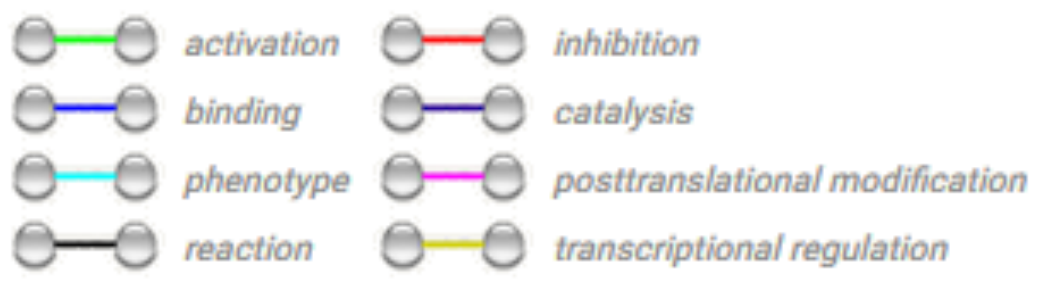

Action effects

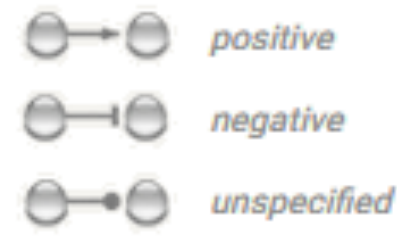

FIGURE 4 Heatmap showing the gene occurrence between genes that belongs to GO BP terms. The yellow color is associated with gene occurrence in the GO Term. The intensity of the color is corresponding to amount of GO BP terms that each gene belongs to

ubiquitously expressed and is present in endomembrane organelles such as vacuoles, lysosomes and endosomes $[21,22]$. The protein secreted by the next gene DKK3, contains two cysteine rich regions and is involved in embryonic development via its connections with the Wnt signaling pathway. The expression of this gene is decreased in a variety of cancer cell lines and it may function as a tumor suppressor gene [23-25]. The conformational regulation of cell growth, immune response, induction and maintenance of pluripotency has also it's place by catalization of Peptidyl-prolyl cis/trans insomerases, which are encoded by the gene PIN1.This enzyme also plays a vital role in the pathogenesis of Alzheimer's disease and similar to DKK3, in some cancers [26,27]. OAS1gene is induced by interferons and encodes a protein that plays a role in other cellular processes such as cell growth, apoptosis, differentiation and gene regulation. A significant role in regulation plays also the NPFFR2 gene, but it regulates reactions in the opioid system. It also functions in pain modulation. NPFF receptors have been implicated in hormonal modulation, regulation of food intake, thermoregulation and nociception [28-30]. The last gene with the highest expression is ATP5B which is now called ATP Synthase F1 Subunit Beta and it's abbreviation changed to ATP5F1B. It takes part in chemical homeostasis by encoding a subunit of mitochondrial ATP synthase. It catalyzes ATP synthesis and utilizes an electrochemical gradient of protons within the inner membrane for the duration of oxidative phosphorylation. Among its related pathways are ATP synthesis by chemiosmotic coupling respiratory electron transport, heat production by uncoupling proteins and purine nucleotides de novo biosynthesis [31,32].

All genes above showed a significantly positive change in expression. Most of them indicated the highest result at the beginning of the cultivation , which has its scientific substantiation. In most cases, these were "-blasting" genes (osteoblasting, myoblasting, etc.), which indicates the tissue formation process that is most intense during the first seven days of culture. There were also genes that affected mainly purely molecular processes, such as activities associated with ATPase or chromatine modulation, which are most active during cell growth and division. This clearly indicates the correctness of our assumption that these genes are subject to the highest expression in the first days of cell culture. 
We will now look at genes that significantly reduced their expression and (when averaged) are arranged as follows, starting with the gene with the most negative expression.

SPP1, which stands for Secreted Phosphoprotein 1 is the gene that encodes protein involved in the attachment of osteoclasts to the mineralized bone matrix. The encoded protein is secreted and compels hydroxyapatite with high similarity. In the cell membrane, the osteoclast vitronectin receptor is found and may be involved in the binding process. Diseases associated with SPP1 include Pediatric Systemic Lupus Erythematosus and Papillary Cystadenocarcinoma. Gene Ontology (GO) notes related to this gene contain extracellular matrix binding and cytokine activity. It is also probably crucial to cell-matrix interaction [33-35]. CCL8 (inorganic cation homeostasis)- is one of several chemokine genes grouped on the q-arm of chromosome 17. Chemokines form a superfamily of secreted proteins. They are involved in inflammatory and immunoregulatory processes. This superfamily is divided into four subfamilies based on the composition of $\mathrm{N}$-terminal cysteine residues of the fully grown peptide. This chemokine is a part of the CC subfamily which is described by two adjacent cysteine residues. This cytokine displays chemotactic activity for lymphocytes, monocytes, eosinophils and basophils. This cytokine may provide to tumor-associated leukocyte infiltration. Gene Ontology (GO) annotations linked to this gene include chemokine and protein kinase activity. An important paralog of this gene is CCL11. Chemotactic factor that may play a role in neoplasia and inflammatory host responses [36-38]. Another member of chemokine superfamily is CXCL2, that encodes secreted proteins involved in inflammatory and immunoregulatory processes. Diseases correlated with CXCL2 include Pneumonia and Peritonitis. A vital paralog of this gene is CXCL1. It is produced by activated neutrophils and monocytes and finally expressed at sites of inflammation $[39,40]$. PTGS2 also known as cyclooxygenase also has its role in the inflammatory reaction. This gene is constitutively expressed in some tissues in physiological conditions, such as the brain, kidney and endothelium and in pathological conditions, such as in cancer. PTGS2 is accountable for production of inflammatory prostaglandins. Although, after averaging, the expression of PTGS2 showed a negative tendency, it should be noted that in the first 7 days it was much stronger than later. This is explained by the fact that up-regulation of this gene is also associated with phenotypic changes, increased cell adhesion, tumor angiogenesis and resistance to apoptosis[41-44].

ATP13A3 and ATP1B1 are representatives of three groups: "cellular divalent inorganic cation homeostasis", "chemical homeostasis" and "homeostatic process". ATP13A3 is a member of the P-type
ATPase family of proteins that carry a variety of cations across membranes [45]. In turn, ATP1B1 gene encodes protein that belongs to the family of $\mathrm{H}+/ \mathrm{K}+$ and $\mathrm{Na}+/ \mathrm{K}+$ ATPases beta chain proteins. They're responsible for determining and maintaining the electrochemical gradients of $\mathrm{K}$ and $\mathrm{Na}$ ions across the plasma membrane. These kind of gradients are essential for sodium-coupled transport of a variety of organic and inorganic molecules, osmoregulation and for electrical excitability of nerve and muscle $[46,47]$. Next is PPARD which encodes a member of the peroxisome proliferator-activated receptor (PPAR) family. The encoded protein is thought to function as nuclear receptor signaling and an integrator of transcriptional repression. An important paralog of this gene is PPARA - a ligand-activated transcription factor. It is the receptor that binds peroxisome proliferators such as fatty acids and hypolipidemic $[48,49]$.

CCL2 and LYN belong to all of the analyzed GO groups. Protein encoded by CCL2 displays chemotactic activity for monocytes and basophils but not for neutrophils or eosinophils. It has been involved in the pathogenesis of diseases described by monocytic infiltrates like rheumatoid arthritis, psoriasis and atherosclerosis [38,50,51]. LYN encodes a tyrosine protein kinase, which is involved in the erythroid differentiation and regulation of mast cell degranulation, and erythroid differentiation. It also plays an important role in hematopoiesis, the regulation of innate and adaptive immune responses, integrin signaling, responses to growth factors and cytokines. It also responses to genotoxic agents and DNA damage [52-54]. The last gene is REL which encodes a protein that belongs to the Rel homology domain/immunoglobulin-like fold, plexin, transcription factor family. Representatives of this family regulate genes involved in inflammation, the immune response, apoptosis and oncogenic processes $[55,56]$.

As revealed above, genes that showed negative expression in the study usually involve lytic processes or responses to pathogenic phenomena. These processes do not occur at such early stages of cell culture or do not take place at all in physiological conditions with no pathogen.

\section{Conclusions}

In summary, the data we have collected show primarily changes in gene expression that occurred in the thirty-day cell culture of oral mucosa tissue. We assume that presented genes can be new gene markers for studied processes. It should be noted, however, that this is not a complete and comprehensive analysis of the subject. To clearly determine the role of these genes, it would be necessary to analyze them in a much larger number of aspects. In addition, there are always several types of cells in samples tested on microarrays, which means different 
results for different percentages of a given type of cells. Finally, the main purpose of the analysis is cell transcripts, which may not always give a complete picture, because there may be a change in the protein product due to processes such as: translation regulation, alternative splicing and post-translational modification. It should also be remembered that our research takes place in vitro, which often gives erroneous results compared to in vivo tests. However, these studies are enough to have a look at how the basic molecular mechanisms work to drive the behavior of oral mucosa cells and can serve as a beginning for clinical trials. Genes have been identified that can be considered as markers for the above-mentioned processes in porcine oral mucosa cells. The differences in expression that we have observed throughout the culture suggest that the homeostasis process itself and the intensity of metabolism occurring in cells is regulated by genes that are involved in "lifespan regulatory mechanisms"[57]. We observed a diverse expression profile of genes involved in 5 gene ontology groups, namely: "cellular divalent inorganic cation homeostasis", "chemical homeostasis", "chemotaxis", "homeostatic process" and "response to organic substance". Genes have been identified that can be considered as markers for the above-mentioned processes in porcine oral mucosa cells. The differences in expression that we have observed throughout the culture suggest that the homeostasis process itself and the intensity of metabolism occurring in cells is regulated by genes that are involved in "lifespan regulatory mechanisms". However, these results need to be confirmed by further analysis on protein level, possibly focused on particular, isolated cell populations found in the oral mucosal tissue.

\section{Corresponding author}

Marta Dyszkiewicz-Konwińska, Department of Biomaterials and Experimental Dentistry, 70 Bukowska St., 60-812 Poznań, Poland and Department of Anatomy, Poznan University of Medical Sciences, 6 Święcickiego St., 60-781 Poznań, Poland, Tel./Fax: +48 61 8546565, e-mail: mdyszkiewicz@ump.edu.pl.

\section{Conflict of interest statement}

The authors declare they have no conflict of interest.

\section{References:}

1. Evans EW. Treating Scars on the Oral Mucosa. Facial Plast Surg Clin North Am. 2017;25:89-97; DOI:10.1016/j.fsc.2016.08.008.

2. Squier CA, Kremer MJ. Biology of oral mucosa and esophagus. J Natl Cancer Inst Monogr. 2001;2001:7-15; DOI:10.1093/oxfordjournals.jncimonographs.a003443.

3. Martin EA (Elizabeth A., Hine R, Oxford University Press. A dictionary of biology. Oxford University Press; 2008

4. Borys S, Khozmi R, Kranc W, Bryja A, Dyszkiewicz-Konwińska M, Jeseta $\mathrm{M}$, et al. Recent findings of the types of programmed cell death. Adv Cell Biol. 2017;5:43-9; DOI:10.1515/acb-2017-0004.

5. Schulz BS, Michel G, Wagner S, Süss R, Beetz A, Peter RU, et al. Increased expression of epidermal IL-8 receptor in psoriasis. Down-regulation by FK-506 in vitro. J Immunol. 1993;151:4399-406;

6. Bigliardi-Qi M, Bigliardi PL, Eberle AN, Büchner S, Rufli T. beta-endorphin stimulates cytokeratin 16 expression and downregulates mu-opiate receptor expression in human epidermis. J Invest Dermatol. 2000;114:527-32; DOI:10.1046/j.1523-1747.2000.00801.x.
7. Grewe M, Gyufko K, Budnik A, Ruzicka T, Olaizola-Horn S, Berneburg M, et al. Interleukin-1 receptors type I and type II are differentially regulated in human keratinocytes by ultraviolet B radiation. J Invest Dermatol. 1996;107:865-70

8. Bryja A, Dyszkiewicz-Konwińska M, Budna J, Ciesiółka S, Kranc W, Borys $\mathrm{S}$, et al. Expression of cell mitotic progression proteins and keratinocyte markers in porcine buccal pouch mucosal cells during short-term, real-time primary culture. J Biol Regul Homeost Agents. n.d.;31:297-309;

9. Bryja A, Dyszkiewicz-Konwińska M, Chachuła A, Ciesiółka S, Kranc W, Bukowska D, et al. Differential expression and distribution of cytokeratins and vimentin in buccal pouch mucosal cells during real-time cell proliferation: research based on a porcine model. J Biol Regul Homeost Agents. n.d.;30:951-60;

10. Huang DW, Sherman BT, Tan Q, Collins JR, Alvord WG, Roayaei J, et al. The DAVID Gene Functional Classification Tool: a novel biological module-centric algorithm to functionally analyze large gene lists. Genome Biol. 2007;8:R183; DOI:10.1186/gb-2007-8-9-r183.

11. Dyszkiewicz-Konwińska M, Nawrocki MJ, Huang Y, Bryja A, Celichowski $\mathrm{P}$, Jankowski M, et al. New gene markers for metabolic processes and homeostasis in porcine buccal pouch mucosa during cells long term-cultivation-a primary culture approach. Int J Mol Sci. 2018;19:1027; DOI:10.3390/ijms19041027.

12. Bryja A, Dyszkiewicz-Konwińska M, Budna J, Kranc W, Chachuła A, Borys $S$, et al. The biomedical aspects of oral mucosal epithelial cell culture in mammals. J Biol Regul Homeost Agents. 2017;31:81-5;

13. Bryja A, Dyszkiewicz-Konwińska M, Budna J, Kranc W, Chachuła A, Ciesiółka S, et al. Kancerogeneza w obrębie błony śluzowej jamy ustnej ssaków w aspekcie badań biomedycznych. Med Weter. 2017;73:82-7 DOI:10.21521/mw.5641.

14. Satterthwaite AB, Burn TC, Le Beau MM, Tenen DG. Structure of the gene encoding CD34, a human hematopoietic stem cell antigen. Genomics. 1992;12:788-94; DOI:10.1016/0888-7543(92)90310-0.

15. San Martin IA, Varela N, Gaete M, Villegas K, Osorio M, Tapia JC, et al. Impaired cell cycle regulation of the osteoblast-related heterodimeric transcription factor Runx2-Cbf $\beta$ in osteosarcoma cells. J Cell Physiol. 2009;221:560-71; DOI:10.1002/jcp.21894.

16. Mitkin NA, Muratova AM, Schwartz AM, Kuprash D V. The A allele of the single-nucleotide polymorphism rs630923 creates a binding site for MEF2C resulting in reduced cxcr5 promoter activity in B-cell lymphoblastic cell lines. Front Immunol. 2016;7:515; DOI:10.3389/ fimmu.2016.00515

17. Nowakowska BA, Obersztyn E, Szymańska K, Bekiesińska-Figatowska M, Xia Z, Ricks CB, et al. Severe mental retardation, seizures, and hypotonia due to deletions of MEF2C. Am J Med Genet Part B Neuropsychiatr Genet. 2010;153:1042-51; DOI:10.1002/ajmg.b.31071.

18. TADA3 transcriptional adaptor 3 [Homo sapiens (human)] - Gene - NCBI n.d.https://www.ncbi.nlm.nih.gov/gene? cmd=Retrieve\&dopt=full_report\&list_uids=10474 (accessed March 2, 2020)

19. Zeng M, Kumar A, Meng G, Gao Q, Dimri G, Wazer D, et al. Human papilloma virus 16 E6 oncoprotein inhibits retinoic $\mathrm{X}$ receptor-mediated transactivation by targeting human ADA3 coactivator. J Biol Chem. 2002;277:45611-8; DOI:10.1074/jbc.M208447200.

20. Gevaert K, Goethals M, Martens L, Van Damme J, Staes A, Thomas GR, et al. Exploring proteomes and analyzing protein processing by mass spectrometric identification of sorted N-terminal peptides. Nat Biotechnol. 2003;21:566-9; DOI:10.1038/nbt810

21. Nishi T, Forgac M. The vacuolar $(\mathrm{H}+)$-ATPases - Nature's most versatile proton pumps. Nat Rev Mol Cell Biol. 2002;3:94-103; DOI:10.1038/ nrm729.

22. Kawasaki-Nishi S, Nishi T, Forgac M. Proton translocation driven by ATP hydrolysis in V-ATPases. FEBS Lett. 2003;545:76-85; DOI:10.1016/ s0014-5793(03)00396-x.

23. Krupnik VE, Sharp JD, Jiang C, Robison K, Chickering TW, Amaravadi L, et al. Functional and structural diversity of the human Dickkopf gene family. Gene. 1999;238:301-13; DOI:10.1016/s0378-1119(99)00365-0.

24. Huang Y, Liu L, Liu A. Dickkopf-1: Current knowledge and related diseases. Life Sci. 2018;209:249-54; DOI:10.1016/j.lfs.2018.08.019.

25. Hamzehzadeh L, Caraglia M, Atkin SL, Sahebkar A. Dickkopf homolog 3 (DKK3): A candidate for detection and treatment of cancers? J Cell Physiol. 2018;233:4595-605; DOI:10.1002/jcp.26313.

26. Cheng CW, Tse E. PIN1 in cell cycle control and cancer. Front Pharmacol. 2018;9DOI:10.3389/fphar.2018.01367.

27. Cheng CW, Tse E. Targeting PIN1 as a Therapeutic Approach for Hepatocellular Carcinoma. Front Cell Dev Biol. 2020;7:369; DOI:10.3389/ fcell.2019.00369.

28. Zhang L, Ip CK, Lee ICJ, Qi Y, Reed F, Karl T, et al. Diet-induced adaptive thermogenesis requires neuropeptide $\mathrm{FF}$ receptor-2 signalling. Nat Commun. 2018;9:4722; DOI:10.1038/s41467-018-06462-0. 
29. Lin YT, Chen JC. Neuropeptide ff modulates neuroendocrine and energy homeostasis through hypothalamic signaling. Chin J Physiol. 2019;62:47-52; DOI:10.4103/CJP.CJP_23_19.

30. Lin YT, Yu YL, Hong WC, Yeh TS, Chen TC, Chen JC. NPFFR2 activates the HPA axis and induces anxiogenic effects in rodents. Int J Mol Sci. 2017;18DOI:10.3390/ijms18081810.

31. Speransky S, Serafini P, Caroli J, Bicciato S, Lippman ME, Bishopric NH. A novel RNA aptamer identifies plasma membrane ATP synthase beta subunit as an early marker and therapeutic target in aggressive cancer. Breast Cancer Res Treat. 2019;176:271-89; DOI:10.1007/ s10549-019-05174-3.

32. Ren L, Ding S, Song Y, Li B, Ramanathan M, Co J, et al. Profiling of rotavirus 3UTR-binding proteins reveals the ATP synthase subunit ATP5B as a host factor that supports late-stage virus replication. J Biol Chem. 2019;294:5993-6006; DOI:10.1074/jbc.RA118.006004.

33. Wang J, Hao F, Fei X, Chen Y. SPP1 functions as an enhancer of cell growth in hepatocellular carcinoma targeted by miR-181c. Am J Transl Res. 2019;11:6924;

34. Zeng B, Zhou M, Wu H, Xiong Z. SPP1 promotes ovarian cancer progression via integrin $\beta 1 /$ FAK/AKT signaling pathway. Onco Targets Ther. 2018;11:1333-43; DOI:10.2147/OTT.S154215.

35. Latoche JD, Ufelle AC, Fazzi F, Ganguly K, Leikauf GD, Fattman CL. Secreted phosphoprotein 1 and sex-specific differences in silica-induced pulmonary fibrosis in mice. Environ Health Perspect. 2016;124:1199-207 DOI:10.1289/ehp.1510335.

36. Sokol CL, Camire RB, Jones MC, Luster AD. The Chemokine Receptor CCR8 Promotes the Migration of Dendritic Cells into the Lymph Node Parenchyma to Initiate the Allergic Immune Response. Immunity. 2018;49:449-463.e6; DOI:10.1016/j.immuni.2018.07.012.

37. Asano K, Takahashi N, Ushiki M, Monya M, Aihara F, Kuboki E, et al. Intestinal CD169 + macrophages initiate mucosal inflammation by secreting CCL8 that recruits inflammatory monocytes. Nat Commun 2015;6DOI:10.1038/ncomms8802.

38. Ge B, Li J, Wei Z, Sun T, Song Y, Khan NU. Functional expression of CCL8 and its interaction with chemokine receptor CCR3. BMC Immunol. 2017;18:54; DOI:10.1186/s12865-017-0237-5.

39. De Filippo K, Dudeck A, Hasenberg M, Nye E, Van Rooijen N, Hartmann K, et al. Mast cell and macrophage chemokines CXCL1/CXCL2 control the early stage of neutrophil recruitment during tissue inflammation. Blood. 2013;121:4930-7; DOI:10.1182/blood-2013-02-486217.

40. Iwasa T, Afroz S, Inoue M, Arakaki R, Oshima M, Raju R, et al. IL-10 and CXCL2 in trigeminal ganglia in neuropathic pain. Neurosci Lett. 2019;703:132-8; DOI:10.1016/j.neulet.2019.03.031.

41. Palliser HK, Kelleher MA, Welsh TN, Zakar T, Hirst JJ. Mechanisms Leading to Increased Risk of Preterm Birth in Growth-Restricted Guinea Pig Pregnancies. Reprod Sci. 2014;21:269-76 DOI:10.1177/1933719113497268.

42. Wang X, Zhao Y, Wang Y, Wang Z, Guan X. Association between a functional variant at PTGS2 gene $3^{\prime}$ UTR and its mRNA expression in lymphoblastoid cell lines. Cell Biol Int. 2013;37:516-9; DOI:10.1002/cbin.10066.

43. Kunzmann AT, Murray LJ, Cardwell CR, McShane CM, McMenamin ÚC, Cantwell MM. PTGS2 (Cyclooxygenase-2) expression and survival among colorectal cancer patients: A systematic review. Cancer Epidemiol Biomarkers Prev. 2013;22:1490-7; DOI:10.1158/1055-9965. EPI-13-0263.

44. Ruan Z, Wang S, Yu W, Deng F. LncRNA MALAT1 aggravates inflammation response through regulating PTGS2 by targeting miR-26b in myocardial ischemia-reperfusion injury. Int J Cardiol. 2019;288:122; DOI:10.1016/j.ijcard.2019.04.015.

45. Madan M, Patel A, Skruber K, Geerts D, Altomare DA, Iv OP. ATP13A3 and caveolin- 1 as potential biomarkers for difluoromethylornithine-based therapies in pancreatic cancers. Am J Cancer Res. 2016;6:1231-52;

46. Wang J, Zhou F, Wang D, Li J, Lu D, Li Q, et al. Interaction of DCF1 with ATP1B1 induces impairment in astrocyte structural plasticity via the P38 signaling pathway. Exp Neurol. 2018;302:214-29; DOI:10.1016/j. expneurol.2018.01.007

47. Shi JL, Fu L, Ang Q, Wang GJ, Zhu J, Wang WD. Overexpression of ATP1B1 predicts an adverse prognosis in cytogenetically normal acute myeloid leukemia. Oncotarget. 2016;7:2585-95; DOI:10.18632/ oncotarget.6226.

48. Zuo X, Xu W, Xu M, Tian R, Moussalli MJ, Mao F, et al. Metastasis regulation by PPARD expression in cancer cells. JCI Insight. 2017;2:e91419; DOI:10.1172/jci.insight.91419.

49. Ye HD, Li YR, Hong QX, Zhou AN, Zhao QL, Xu LM, et al. Positive association between PPARD rs2016520 polymorphism and coronary heart disease in a Han Chinese population. Genet Mol Res. 2015;14:6350-9, DOI:10.4238/2015.June.11.10.
50. Charpidou A, Kotteas E, Gaga M. Towards precision medicine: CCL2, another brick in the wall? Eur Respir J. 2019;53DOI:10.1183/13993003.02327-2018.

51. Conti I, Rollins BJ. CCL2 (monocyte chemoattractant protein-1) and cancer. Semin Cancer Biol. 2004;14:149-54; DOI:10.1016/j. semcancer.2003.10.009.

52. Brodie EJ, Infantino S, Low MSY, Tarlinton DM. Lyn, lupus, and (B) lymphocytes, a lesson on the critical balance of kinase signaling in immunity. Front Immunol. 2018;9:401; DOI:10.3389/fimmu.2018.00401.

53. Blasioli J, Goodnow CC. Lyn/CD22/SHP-1 and their importance in autoimmunity. Curr Dir Autoimmun. 2002;5:151-60; DOI:10.1159/000060551.

54. Hibbs ML, Dunn AR. Lyn, a src-like tyrosine kinase. Int J Biochem Cell Biol. 1997;29:397-400; DOI:10.1016/s1357-2725(96)00104-5.

55. Rushlow C, Warrior R. The rel family of proteins. BioEssays. 1992;14:8995; DOI:10.1002/bies.950140204.

56. Nolan GP, Baltimore D. The inhibitory ankyrin and activator Rel proteins. Curr Opin Genet Dev. 1992;2:211-20; DOI:10.1016/ s0959-437x(05)80276-x.

57. Dyszkiewicz-Konwińska M, Nawrocki M, Huang Y, Bryja A, Celichowsk P, Jankowski M, et al. New Gene Markers for Metabolic Processes and Homeostasis in Porcine Buccal Pouch Mucosa during Cells Long Term-Cultivation-A Primary Culture Approach. Int J Mol Sci. 2018;19:1027; DOI:10.3390/ijms19041027. 